\title{
DISCIPLINARY PUNISHMENTS IN MILITARY POLICE AS CONSEQUENCE OF THE HARMFUL USE OF PSYCHOACTIVE SUBSTANCES
}

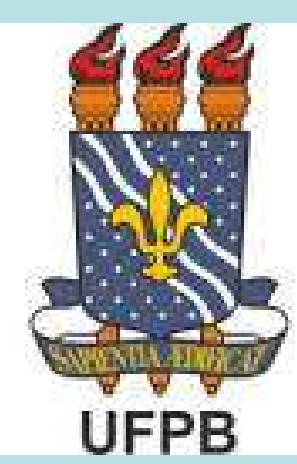

$\underline{\text { l. Frazão }}^{1}$, P. Amorim Neto Dias de ${ }^{1}$, F. Silva Pereira da ${ }^{2}$, A.L. Silva Medeiros Araújo da ${ }^{1}$, S. Vasconcelos Cordeiro ${ }^{3}$, V. França

${ }^{1}$ Federal University of Pernambuco, Nursing Department, Recife, Brazil. ${ }^{2}$ Mauricio de Nassau University Center, Nursing and Mental Health, Recife, Brazil. ${ }^{3}$ Federal University of Paraiba, Nursing, João Pessoa, Brazil.

\section{Background}

The decrease in behavioral performance in service may be related to the high prevalence of disciplinary punishments evidenced among military police officers in treatment for the harmful use of psychoactive substances.

\section{Objective}

To analyze the repercussions of the application of disciplinary punishments in military police as consequence of the harmful use of psychoactive substances.

\section{Materials and Methods}

This is a cross-sectional study, with a quantitative approach, developed at the Center for Chemical Addiction of the Military Police of Pernambuco, Brazil. The sample consisted of 251 military police records. Data analysis was performed using descriptive statistics, using association test (chi-square or Fisher's exact test) and Poisson regression with robust variance.

\section{Results and Conclusions}

The greatest proportion of police were male (99.2\%), married (70.1\%), age between 41 and $50(50.2 \%)$, high school (48.2\%) and income between 767 and income about 1279 dollars/month (93.2\%). Negative consequences in the evaluation of military performance was verified by the high prevalence of disciplinary punishment (76.5\%); imprisonment was the most prevalent punishment $(49.4 \%)$ and alcohol the most prevalent substance $(79.7 \%)$. Regarding the multivariate analysis, the disciplinary punishment showed a statistically significant association with the instruction level. The enlisted grades had the highest prevalence risk (98.4\%). In relation to the age, the police under 50 years obtained greater risk to receive disciplinary punishment.

Substance use according to socio-demographic data of military police. Pernambuco, Brazil, 2018.

\section{Poisson model adjustment for the risk of disciplinary punishment according to age and police rank of military police. Pernambuco, Brazil, 2018.}

\section{Variable}

Psicoative Substance

\begin{tabular}{|c|c|c|c|c|}
\hline Variable & Alcohol & Others drugs & $\begin{array}{c}\text { Alcohol } \\
+ \\
\text { Others }\end{array}$ & $p$-valt \\
\hline \multicolumn{5}{|l|}{ Marital Status } \\
\hline Married/engaged & $150(82.4 \%)$ & $12(6,6 \%)$ & $20(11,0 \%)$ & \\
\hline Single & $22(64,7 \%)$ & $2(5,9 \%)$ & $10(29,4 \%)$ & $0,027^{2}$ \\
\hline Divorced/widowed & $28(80 \%)$ & 0 & $7(20 \%)$ & \\
\hline \multicolumn{5}{|l|}{ Age (Years) } \\
\hline $20+30$ & $16(44,4 \%)$ & $8(22,2 \%)$ & $12(33,3 \%)$ & \\
\hline $30 \vdash-40$ & $61(77,2 \%)$ & $4(5,1)$ & $14(17,7 \%)$ & \\
\hline $40-50$ & $113(89,7 \%)$ & $2(1,6 \%)$ & $11(8,7 \%)$ & $<0,001^{2}$ \\
\hline$>50$ & $10(100 \%)$ & 0 & 0 & \\
\hline \multicolumn{5}{|l|}{ Educational Stages } \\
\hline $\begin{array}{l}\text { Until primary school } \\
\text { (<=8 years) }\end{array}$ & $102(91,1 \%)$ & 0 & $10(8,9 \%)$ & \\
\hline $\begin{array}{l}\text { Secondary school (>8-11 } \\
\text { years) }\end{array}$ & $89(73,6 \%)$ & $9(7,4 \%)$ & $23(19,0 \%)$ & ) $<0,001^{2}$ \\
\hline $\begin{array}{l}\text { Higher education (>15 } \\
\text { years) }\end{array}$ & $9(50,0 \%)$ & $5(25,0 \%)$ & $4(25,0 \%)$ & \\
\hline \multicolumn{5}{|l|}{ Years of Police Work } \\
\hline $1 \vdash 10$ & $26(46,4 \%)$ & $11(19,6 \%)$ & $19(33,9 \%)$ & \\
\hline $10 \vdash 20$ & $45(84,9 \%)$ & $1(1,9 \%)$ & $7(13,2 \%)$ & \\
\hline $20 \mid-30$ & $121(90,3 \%)$ & $2(1,5 \%)$ & $11(8,2 \%)$ & $<0,001^{2}$ \\
\hline$>30$ & $8(100 \%)$ & 0 & 0 & \\
\hline
\end{tabular}

\begin{tabular}{c|c|c|c|}
\hline Variable & \multicolumn{3}{|c}{ Adjust } \\
\hline $\begin{array}{c}\text { Age (years) } \\
<50\end{array}$ & 0,75 & $\begin{array}{c}-0,47 \mathrm{a}- \\
0,11\end{array}$ & 0.002 \\
\hline \begin{tabular}{c}
$\mathrm{Cl}(95 \%)$ \\
\hline Military Rank
\end{tabular} & 1 & - & - \\
\hline $\begin{array}{c}\text { Non-value } \\
\text { officers } \\
\text { officed }\end{array}$ & 0,69 & $\begin{array}{c}-0,50 \mathrm{a}- \\
0,26\end{array}$ & $<0,001$ \\
\hline Officer & 1 & - & - \\
\hline
\end{tabular}

$\mathrm{PR}=$ prevalence ratio; $\mathrm{Cl}=$ confidence interval.

${ }^{1}$ Qui-Square, ${ }^{2}$ Fisher's exact test.

References: AKBARI, J.; AKBARI, R.; SHAKERIAN, M.; MAHAKI, B. Job demand-control and job stress at work: A cross-sectional study among prison staff. J Educ Health Promot, Iran, v.6, n.15, abr 2017. DOI: 10.4103/jehp.jehp_68_14 . ASCARI, R. A.; DUMKE, M.; DACOL, P. M.; MAUS-JUNIOR, S.; SÁ, C. A.; LAUTERT, L. Prevalência de risco para Síndrome de Burnout em policiais militares. Cogitare Enferm, São Paulo, v.21, n.2, p.01-10, 2016. DOI: 10.5380/ce.v21i2.44610. BOUDOUKHA, A. H.; PRZYGODZKILIONET, N; HAUTEKEETE, M. Traumatic events and early maladaptive schemas (EMS): Prison guard psychological vulnerability. Eur Rev ApplPsychol, França, v.66, n.4, p.181-7, Jul 2016. DOI: 10.1016/j.erap.2011.05.004. BRASIL. Presidência da República. Secretaria Nacional de Políticas sobre Drogas. Relatório brasileiro sobre drogas / Secretaria Nacional de Políticas sobre Drogas; IME USP; organizadores Paulina do Carmo Arruda Vieira Duarte, Vladimir de Andrade Stempliuk e Lúcia Pereira Barroso. Brasília: SENAD, 2009. COSTA, S. H. N.; CUNHA, L. C.; PUCCI, Y. L. L.; OLIVEIRA, F. G. F.; SOUZA, C. G.; MESQUITA, G. A. Survey on the use of psychotropic drugs by twelve military police units in the municipalities of Goiânia and Aparecida de Goiânia, state of Goiás, Brazil. Rev Bras Psiquiatr., Goiás, v.32, n.4, p. 389-95, 2010. DOI: http://dx.doi.org/10.1590/S1516-44462010005000023. 